\title{
Markov Chain Monte Carlo methods applied to measuring the fine structure constant from quasar spectroscopy
}

\author{
Julian King ${ }^{1}$, Daniel Mortlock ${ }^{2}$, John Webb $^{1}$ and Michael Murphy ${ }^{3}$ \\ ${ }^{1}$ Department of Astronomy, University of New South Wales, \\ Sydney, New South Wales, 2052, Australia \\ email: jking.phys@gmail.com \\ ${ }^{2}$ Astrophysics Group, Blackett Laboratory, Prince Consort Road, Imperial College London, \\ London SW7 2AZ, U.K. \\ ${ }^{3}$ Centre for Astrophysics and Supercomputing, Swinburne University of Technology, \\ Victoria, 3122, Australia
}

\begin{abstract}
Recent attempts to constrain cosmological variation in the fine structure constant, $\alpha$, using quasar absorption lines have yielded two statistical samples which initially appear to be inconsistent. One of these samples was subsequently demonstrated to not pass consistency tests; it appears that the optimisation algorithm used to fit the model to the spectra failed. Nevertheless, the results of the other hinge on the robustness of the spectral fitting program VPFIT, which has been tested through simulation but not through direct exploration of the likelihood function. We present the application of Markov Chain Monte Carlo (MCMC) methods to this problem, and demonstrate that VPFIT produces similar values and uncertainties for $\Delta \alpha / \alpha$, the fractional change in the fine structure constant, as our MCMC algorithm, and thus that VPFIT is reliable.
\end{abstract}

Keywords. Atomic processes - Methods: numerical - Methods: statistical - Quasars: absorption lines - Quasars: individual: LBQS 2206-1958 - Quasars: individual: LBQS 0013-0029 Quasars: individual: Q 0551-366 - Cosmology: observations

Recent years have seen sustained interest in attempting to determine the value of the fine structure constant, $\alpha$ in the early universe using quasar absorption lines. Differing atomic/ionic transitions have different sensitivities to $\alpha$, and thus by fitting Voigt profiles to the observed profiles of quasar absorption systems, we can mesure $\Delta \alpha / \alpha=\left(\alpha_{z}-\alpha_{0}\right) / \alpha_{0}$, where $\alpha_{z}$ is the value of $\alpha$ at redshift $z$ and $\alpha_{0}$ is the laboratory value. Murphy et al. (2004) have found that $\Delta \alpha / \alpha=(-0.57 \pm 0.11) \times 10^{-5}$ from 143 quasar absorption systems, whereas Chand et al. (2004) reported $\Delta \alpha / \alpha=(-0.06 \pm 0.06) \times 10^{-5}$ from 23 measurements. However, Murphy et al. $(2008)$ demonstrate that the analysis of Chand et al. suffers significant flaws which render both the estimate and statistical precision for $\Delta \alpha / \alpha$ of Chand et al. unreliable.

We apply Markov Chain Monte Carlo (MCMC) methods to investigate the $z=1.018$, $z=2.029$ and $z=1.748$ absorption systems toward quasars LBQS 2206-1958, LBQS 0013-0029 and Q 0551-366 respectively. In particular, we confirm for these cases that the spectral fitting program VPFIT, used by Murphy et al. (2004), produces good estimates of $\Delta \alpha / \alpha$ and appropriate statistical uncertainties, and thus we regard the results of Murphy et al. (2004) as robust.

\section{References}

Chand, H., Srianand, R., Petitjean, P., \& Aracil, B. 2004, A\& A, 417, 853

Murphy, M. T., Flambaum, V. V., Webb, J. K., et al. 2004, in: S. G. Karshenboim \& E. Peik (eds.), Astrophysics, Clocks and Fundamental Constants, Lecture Notes in Physics, Berlin Springer Verlag, Vol. 648 (Berlin: Springer Verlag), p. 131

Murphy, M. T., Webb, J. K., \& Flambaum, V. V. 2008, MNRAS, 384, 1053 